\title{
Estoques e frações da matéria orgânica do solo sob os sistemas plantio direto e convencional de repolho
}

\author{
Gabriel Bressiani Melo(1), Marcos Gervasio Pereira(2), Adriano Perin ${ }^{(1)}$, \\ Roni Fernandes Guareschi ${ }^{(2)}$ e Paula Fernanda Chaves Soares ${ }^{(2)}$
}

\begin{abstract}
(1)Instituto Federal Goiano, Departamento de Fitotecnia, Campus Rio Verde, Avenida Sul Goiânia, Km 1, Zona Rural, CEP $75901-970$ Rio Verde, GO, Brasil. E-mail: gabremelo@gmail.com, perinrj@yahoo.com.br (2)Universidade Federal Rural do Rio de Janeiro, Departamento de Solos, BR-465, Km 7, CEP 23890-000 Seropédica, RJ, Brasil. E-mail: mgervasiopereira01@gmail.com, guareschiecotarelli@hotmail.com, pfernanda07@gmail.com
\end{abstract}

Resumo - O objetivo deste trabalho foi avaliar as frações húmicas, granulométricas e oxidáveis da matéria orgânica do solo, o conteúdo de matéria orgânica leve no solo, e os teores e estoques de $\mathrm{C}$ e $\mathrm{N}$ em Latossolo Vermelho, manejado pelos sistemas plantio direto e convencional, para o cultivo de repolho. Áreas de pastagem e de cerrado nativo foram utilizadas como controle. As amostras foram coletadas em 2014, às profundidades $0,0-0,05$ e $0,05-0,10 \mathrm{~m}$, após um ciclo de cultivo do repolho sob plantio direto e convencional, em área com longo histórico de cultivo convencional de hortaliças. O sistema plantio direto aumenta os teores de matéria orgânica leve, $\mathrm{C}$ e $\mathrm{N}$, assim como os estoques de $\mathrm{C}$ e os valores de $\mathrm{C}$ orgânico particulado, de $\mathrm{C}$ orgânico associado a minerais, e de frações húmicas e oxidáveis da matéria orgânica. O sistema plantio direto melhora a qualidade do solo, em comparação ao plantio convencional, além de proporcionar, para a maioria das variáveis analisadas, maiores valores do que os observados em área de pastagem e valores semelhantes aos da área sob cerrado nativo.

Termos para indexação: compartimentos da matéria orgânica, dinâmica da matéria orgânica, manejo do solo, matéria orgânica leve, plantio direto de hortaliças.

\section{Storage and fractions of soil organic matter under no-tillage and conventional planting systems of cabbage}

\begin{abstract}
The objective of this work was to evaluate the humic, granulometric, and oxidizable fractions of soil organic matter, the light organic matter content in the soil, and $\mathrm{C}$ and $\mathrm{N}$ contents and storage in an Oxisol under no-tillage and conventional tillage systems for cabbage cultivation. Pasture areas and native cerrado soil were used as a control. The samples were collected in 2014 at the $0.0-0.05$ and $0.05-0.10-\mathrm{m}$ soil depths, after one cabbage crop cycle under no-tillage and conventional tillage systems, in an area with a long history of conventional tillage for vegetable crops. The no-tillage system increases the contents of light organic matter, $\mathrm{C}$ and $\mathrm{N}$, as well as $\mathrm{C}$ storage and the values of particulate organic $\mathrm{C}$, organic $\mathrm{C}$ associated with minerals, and the humic and oxidizable fractions of organic matter. The no-tillage system improves soil quality, in comparison with conventional tillage, besides providing, for most of the variables, higher values than those observed in the pasture area and similar values to those of the area under native cerrado.

Index terms: organic matter compartments, dynamics of organic matter, soil management, light organic matter, no-tillage for vegetables.
\end{abstract}

\section{Introdução}

O intenso preparo e revolvimento do solo em cultivos de hortaliça pode, com o tempo, reduzir a quantidade e a qualidade da matéria orgânica do solo (Souza et al., 2014a; Ramos et al., 2015). Portanto, o interesse pelo uso de sistemas conservacionistas para a produção de hortaliças tem crescido no país, com reflexos positivos para a qualidade do solo (Souza \& Guimarães, 2013; Souza et al., 2014a) e produtividade das culturas (Galvão et al., 2013; Neves et al., 2014). Assim, o cultivo de hortaliças em sistema plantio direto (SPD) tem-se difundido no Brasil e no mundo, com forte tendência ao crescimento em sua adoção (Souza et al., 2014a).

É notório que, em razão do tempo de implantação, o SPD em culturas anuais promove diversos benefícios ao solo, tais como o aumento do teor de C e N (Siqueira Neto et al., 2010; Guareschi et al., 2012a; Pereira et al., 
2013), bem como melhorias dos atributos físicos e químicos (Silva et al., 2008; Siqueira Neto et al., 2010; Guareschi et al., 2012a, 2013; Beutler et al., 2015) e biológicos (Leite et al., 2010; Pereira et al., 2013). No entanto, ainda são poucos os estudos que avaliam a dinâmica da matéria orgânica e de suas frações, ou os benefícios destas, em solos sob sistemas plantio direto de hortaliças (SPDH) no cerrado (Souza et al., 2014a, 2014b).

Ao avaliar sistemas orgânicos de produção de hortaliças sob SPD, em diferentes classes de solo, no Estado do Espírito Santo, Santos et al. (2012) constataram que esse manejo elevou o teor de matéria orgânica. De maneira similar, Souza et al. (2014a) verificaram que a adoção de SPD no cultivo de repolho reduziu em $90 \%$ as taxas de perda de água, em comparação ao plantio convencional. Em outro estudo, Souza et al. (2014b) relatam que o SPDH favorece o aumento do C orgânico total (COT) e das frações mais lábeis e oxidáveis da matéria orgânica, principalmente na camada superficial do solo, em comparação ao plantio convencional (SPC); no entanto, segundo os autores, o SPDH somente promoveu o aumento nos teores de humina, na área de SPD com hortaliças. No Estado do Rio de Janeiro, Loss et al. (2009, 2010a, 2010b), ao comparar um SPD com rotação entre beringela e milho e um SPC com rotação entre milho e feijão, em Argissolo, relataram maiores teores - no SPD - de matéria orgânica leve (MOL), COT, $\mathrm{N}$ do solo, $\mathrm{C}$ orgânico particulado (COp), C orgânico associado aos minerais (Coam), e maiores valores de frações húmicas e frações oxidáveis da MOS (graus de oxidação F2, F3 e F4).

Porém, Bianchini (2013) reporta resultados contraditórios, em que os teores de $\mathrm{N}$ e COT do solo, bem como os de COp, após três anos de implantação do SPDH, não foram influenciados pelos sistemas de manejo, em Nitossolo Vermelho cultivado com abobrinha de tronco. Pauletti (2012), ao avaliar a cultura da alface, em Latossolo Vermelho, sob SPDH com um ano de implantação e diferentes plantas de cobertura, também não verificou alterações em COT e densidade do solo (Ds), tendo observado apenas maiores teores de COT entre as camadas do solo. Ao avaliar a evolução da fertilidade do solo após quatro pré-cultivos de plantas de cobertura, na adoção de SPDH para a produção de repolho, Souza \& Guimarães (2013) também não constataram alteração dos teores de COT.
Esses resultados contrastantes podem ser decorrentes de diversos fatores, tais como manejo e condições edafoclimáticas distintas, diferentes aportes de resíduos orgânicos e avaliações em diferentes fases de evolução do SPDH. Isso reforça a ideia de que mais estudos precisam ser realizados no Brasil, com o intuito de isolar estas fontes de variação e determinar o real efeito do SPDH sobre as frações da MOS, bem como para propor possíveis melhorias no desenvolvimento desse sistema de manejo do solo.

Assim, a avaliação de alguns atributos do solo sob SPDH, tais como, COT, N, matéria orgânica leve (MOL), frações químicas, granulométricas e oxidáveis da MOS, é de grande importância, uma vez que eles têm apresentado grande sensibilidade ao tipo de manejo adotado no Cerrado (Pereira et al., 2010; Loss et al., 2012; Guareschi et al., 2012a, 2013; Beutler et al., 2015).

O objetivo deste trabalho foi avaliar o teor e os estoques de $\mathrm{C}$ e $\mathrm{N}$ em um Latossolo Vermelho, bem como determinar os valores da matéria orgânica leve e das frações húmicas, granulométricas e oxidáveis da MOS em áreas de SPDH e SPC, no sudoeste de Goiás.

\section{Material e Métodos}

As áreas de estudo estão localizadas no Setor de Olericultura do Instituto Federal Goiano, Campus Rio Verde, no sudoeste de Goiás ( $17^{\circ} 47^{\prime} 53^{\prime \prime S}$ e $51^{\circ} 55^{\prime} 53^{\prime \prime} \mathrm{W}$, a $743 \mathrm{~m}$ de altitude). O solo das áreas foi classificado como Latossolo Vermelho eutroférrico típico de textura média.

Avaliaram-se duas áreas de manejo, além de duas áreas de referência (pastagem e cerrado nativo), num total de quatro sistemas diferenciados. As áreas avaliadas compreenderam: SPDH, cultivo de repolho em SPD (fase inicial de implantação); SPC, cultivo de repolho em sistema de plantio convencional; PA, pastagem cultivada com Urochloa decumbens (syn. Brachiaria decumbens); e CE, cerrado nativo (stricto sensu).

As áreas cultivadas com repolho sob SPC e SPD apresentaram o seguinte histórico: vegetação original de cerrado nativo, que foi derrubado para implantação de Urochloa decumbens, a qual foi explorada por mais de 10 anos. A partir de 1975, a área de pastagem foi substituída pelo cultivo de hortaliças sob SPC, com preparo do solo com o emprego de enxada rotativa. 
O SPD foi adotado em parte da área, em 2013, com o plantio de milho para a formação de palha $(80 \mathrm{mil}$ plantas ha-1), com produção de $16 \mathrm{Mg} \mathrm{ha}^{-1}$ de matéria seca.

Em abril de 2014, selecionaram-se duas áreas a para produção de repolho, uma sob SPD e outra sob SPC. Para a área de SPD, a dessecação da cobertura vegetal foi feita com o herbicida glifosato, à dose de $960 \mathrm{~g} \mathrm{ha}^{-1}$ i.a, e, para a área de SPC, realizou-se capina manual. Sete dias após a dessecação ou a capina das áreas, foi feito o transplante manual das mudas, no espaçamento $0,40 \times 0,40 \mathrm{~m}$, com população final de 62.500 plantas $\mathrm{ha}^{-1}$. A área total utilizada em cada sistema de cultivo foi de 18,10x26,30 m $\left(476,03 \mathrm{~m}^{2}\right)$. Essas áreas receberam irrigação três vezes por semana, durante 12 semanas, com $6 \mathrm{~mm}$ de lâmina d'água por dia, o que equivale a $216 \mathrm{~mm}$ durante todo o ciclo da cultura. As adubações seguiram as recomendações técnicas para a região e foram as mesmas utilizadas em ambos os sistemas, com aplicação de $1.050,36 \mathrm{~kg}$ ha $^{-1}$ de 0-20-20 (N- $\left.\mathrm{P}_{2} \mathrm{O}_{5}-\mathrm{K}_{2} \mathrm{O}\right)$ e $525,18 \mathrm{~kg} \mathrm{ha}^{-1}$ de ureia em 29 de maio e em 4 de julho de 2014. O controle das plantas espontâneas na área de plantio convencional foi feito manualmente, aos 22 dias após o transplante do repolho. $\mathrm{Na}$ área com SPD, não houve necessidade de capina. A colheita do repolho foi realizada aos 82 dias após o transplante. A produção de matéria fresca foi de 98,2 e 67,3 $\mathrm{Mg} \mathrm{ha}^{-1}$, nas áreas de SPD e SPC, respectivamente; e o diâmetro de cabeças de 21,5 e $17,14 \mathrm{~cm}$. Portanto, SPD aumentou a produção e a qualidade do repolho, em comparação ao SPC.

A área de cerrado stricto sensu faz parte da reserva do Instituo Federal Goiano e tem cerca de 20 ha. A área de $U$. decumbens vem sendo manejada com taxa de lotação aproximada de 1,5 unidade animal por ha, sem adubações ou correção do solo nos últimos 15 anos. Essas áreas encontram-se sob as mesmas condições edafoclimáticas e são adjacentes às áreas de SPC e SPD; assim, elas foram tomadas como referência para a comparação dos resultados dos sistemas de manejo do solo avaliados.

Em cada área de estudo (SPD, SPC, PA e CE), demarcaram-se quatro glebas representativas e, em cada uma delas, realizou-se a coleta de amostras indeformadas, por meio de um anel volumétrico (Donagemma et al., 2011), às profundidades de $0,0-0,05$ e $0,05-0,10 \mathrm{~m}$. Também foram coletadas, de foram aleatória nessas profundidades, quatro amostras deformadas compostas, cada uma com 10 amostras simples. As amostras foram secas ao ar, destorroadas e passadas por peneira de $2 \mathrm{~mm}$ de malha, para obtenção da terra fina seca ao ar (TFSA), que foi utilizada para a maioria das análises químicas (Donagemma et al., 2011) (Tabela 1). A Ds foi determinada com o método do anel volumétrico (Donagemma et al., 2011).

A matéria orgânica leve (MOL) foi avaliada pelo método da flotação em água (Anderson \& Ingram, 1989), e seus teores de $C$ foram determinados segundo Yeomans \& Bremner (1988). A determinação do COT foi realizada pelo método de oxidação por via úmida, com aquecimento externo. $\mathrm{O} \mathrm{N}$ total do solo foi quantificado por meio de digestão sulfúrica e destilação Kjeldahl. A partir dos teores de COT e N e dos valores da Ds, calcularam-se os estoques de $\mathrm{C}$ (EstC) e N (EstN) pelo método de massa equivalente (Sisti et al., 2004).

O fracionamento granulométrico da MOS foi feito conforme Cambardella \& Elliott (1993), para obtenção

Tabela 1. Resultados das análises química e textural do solo.

\begin{tabular}{|c|c|c|c|c|c|c|c|c|c|c|c|c|}
\hline Área & $\mathrm{pH}$ & $\mathrm{P}$ & $\mathrm{Ca}$ & $\mathrm{Mg}$ & $\mathrm{K}$ & $\mathrm{Al}$ & $\mathrm{H}+\mathrm{Al}$ & $\mathrm{T}$ & \multirow{2}{*}{$\begin{array}{c}\mathrm{V} \\
(\%)\end{array}$} & Argila & Silte & Areia \\
\hline & $\mathrm{H}_{2} \mathrm{O}$ & $\left(\mathrm{mg} \mathrm{kg}^{-1}\right)$ & ------- & ------ & $--(\mathrm{cn}$ & $\left.g^{-1}\right)--$ & ------- & --------- & & ---------. & $\left(\mathrm{g} \mathrm{kg}^{-1}\right)$ & -------- \\
\hline & \multicolumn{12}{|c|}{ Camada $0,0-0,05 \mathrm{~m}$} \\
\hline SPDH & 5,03 & 98,91 & 5,15 & 1,83 & 0,88 & 0,05 & 8,46 & 16,37 & 48 & 392,0 & 432,0 & 176,0 \\
\hline SPC & 4,84 & 46,21 & 3,78 & 2,40 & 0,89 & 0,03 & 9,20 & 16,30 & 44 & 378,0 & 418,0 & 204,0 \\
\hline PA & 5,25 & 0,88 & 2,90 & 2,75 & 0,62 & 0,00 & 6,15 & 12,42 & 51 & 390,0 & 434,0 & 176,0 \\
\hline \multirow[t]{2}{*}{$\mathrm{CE}$} & 5,23 & 3,23 & 3,18 & 2,30 & 0,85 & 0,03 & 8,99 & 15,35 & 41 & 401,0 & 407,0 & 192,0 \\
\hline & \multicolumn{12}{|c|}{ Camada $0,05-0,10 \mathrm{~m}$} \\
\hline SPDH & 4,66 & 69,26 & 3,5 & 2,38 & 1,15 & 0,04 & 9,82 & 16,89 & 42 & 406,0 & 396,0 & 198,0 \\
\hline $\mathrm{SPC}$ & 4,68 & 50,57 & 4,38 & 1,83 & 0,79 & 0,08 & 8,66 & 15,74 & 45 & 402,0 & 407,0 & 191,0 \\
\hline $\mathrm{PA}$ & 5,17 & 0,25 & 2,15 & 3,88 & 0,63 & 0 & 6,11 & 12,77 & 52 & 393,0 & 396,0 & 211,0 \\
\hline $\mathrm{CE}$ & 5,15 & 2,25 & 1,85 & 4,05 & 0,9 & 0,09 & 7,96 & 14,85 & 46 & 437,0 & 371,0 & 192,0 \\
\hline
\end{tabular}

SPDH, sistema plantio direto de hortaliças; SPC, sistema de plantio convencional; PA, pastagem de Urochloa decumbens; e CE, área de cerrado nativo. 
do COp. Utilizaram-se $20 \mathrm{~g}$ de TFSA e $60 \mathrm{~mL}$ de solução de hexametafosfato de sódio $\left(5 \mathrm{~g} \mathrm{~L}^{-1}\right)$, com agitação por 15 horas em agitador horizontal. Em seguida, a suspensão foi passada por peneira de $53 \mu \mathrm{m}$. $\mathrm{O}$ material retido na peneira, o próprio $\mathrm{COp}$, foi seco em estufa a $50^{\circ} \mathrm{C}$, para determinação da massa, e moído em gral de porcelana para análise quanto ao teor de C, por oxidação por via úmida (Yeomans \& Bremner, 1988). O Coam foi obtido a partir da diferença entre $C$ total e COp.

Para o fracionamento químico da MOS, utilizou-se a técnica de solubilidade diferencial, modificada por Benites et al. (2003). As frações da matéria orgânica foram identificadas como frações ácido fúlvico (AF), ácido húmico $(\mathrm{AH})$ e humina (HUM), conforme os termos estabelecidos pela Sociedade Internacional de Substâncias Húmicas.

$\mathrm{O}$ fracionamento do $\mathrm{C}$ por graus de oxidação foi feito conforme Chan et al. (2001). Amostras de 0,3 g de solo foram acondicionadas em frascos de Erlenmeyer de $250 \mathrm{~mL}$, aos quais se adicionaram $10 \mathrm{~mL}$ de $\mathrm{K}_{2} \mathrm{Cr}_{2} \mathrm{O}_{7}$ a $0,167 \mathrm{~mol} \mathrm{~L}^{-1}$ e quantidades de $\mathrm{H}_{2} \mathrm{SO}_{4}$ p.a. correspondentes às concentrações de 3, 6, 9 e $12 \mathrm{~mol} \mathrm{~L}^{-1}$. A oxidação foi realizada sem fonte externa de calor, e a titulação dos extratos foi feita com uma solução de $\mathrm{Fe}\left(\mathrm{NH}_{4}\right)_{2}\left(\mathrm{SO}_{4}\right)_{2} 6 \mathrm{H}_{2} \mathrm{O} 0,4 \mathrm{~mol} \mathrm{~L}^{-1}$ (sal de Mohr), com uso da fenantrolina $\left(\mathrm{C}_{12} \mathrm{H}_{8} \mathrm{~N}_{2} \mathrm{H}_{2} \mathrm{O}\right)$ como indicador. $\mathrm{O}$ fracionamento do $\mathrm{C}$ produziu quatro frações, com graus decrescentes de oxidação: $\mathrm{F} 1$, fração 1 , com $\mathrm{C}$ oxidado por $\mathrm{K}_{2} \mathrm{Cr}_{2} \mathrm{O}_{7}$ em meio ácido a $3 \mathrm{~mol} \mathrm{~L}^{-1}$ de $\mathrm{H}_{2} \mathrm{SO}_{4} ; \mathrm{F} 2$, fração 2 , obtida pela diferença do C oxidado a 6 e a $3 \mathrm{~mol} \mathrm{~L}^{-1}$ de $\mathrm{H}_{2} \mathrm{SO}_{4} ; \mathrm{F} 3$, fração 3, obtida pela diferença do C oxidado a 9 e a $6 \mathrm{~mol} \mathrm{~L}^{-1}$; e $\mathrm{F} 4$, fração 4 , obtida pela diferença do $\mathrm{C}$ oxidado a 12 e a $9 \mathrm{~mol} \mathrm{~L}^{-1}$.

Utilizou-se um delineamento inteiramente casualizado, em arranjo de parcelas subdivididas $4 \times 2$, com quatro áreas, duas profundidades e quatro pseudorrepetições. Os resultados foram submetidos à análise de normalidade da distribuição (teste de Lilliefors) e de homogeneidade das variâncias (teste de Cochran). Quando atenderam às pressuposições de normalidade e homogeneidade, as médias foram comparadas pelo teste $\mathrm{t}$ de Bonferroni, a $5 \%$ de probabilidade.

\section{Resultados e Discussão}

As áreas de SPDH e CE apresentaram os maiores teores de MOL, COT, EstC $(0,0-0,10 \mathrm{~m})$ e N $(0,0-$
$0,05 \mathrm{~m})$, em comparação às áreas de SPC e PA (Tabela 2). Nas áreas de CE e SPDH, os valores de MOL, COT e $\mathrm{N}$ foram maiores na camada superficial $(0,0-0,05 \mathrm{~m})$. Não houve diferença significativa entre as áreas quanto ao teor de $\mathrm{N}(0,05-0,10 \mathrm{~m})$ e de EstN $(0,0-0,10 \mathrm{~m})$.

Tabela 2. Atributos relacionados à matéria orgânica do solo, em áreas sob plantio direto de hortaliças (SPDH) e sob plantio convencional (SPC), cultivadas com repolho, pastagem (PA) e sob cerrado nativo (CE), em duas profundidades ${ }^{(1)}$.

\begin{tabular}{|c|c|c|}
\hline \multirow[t]{2}{*}{ Área } & \multicolumn{2}{|c|}{ Profundidade } \\
\hline & $0,0-0,05 \mathrm{~m}$ & $0,05-0,10 \mathrm{~m}$ \\
\hline & \multicolumn{2}{|c|}{$\operatorname{MOL}\left(\mathrm{g} \mathrm{kg}^{-1}\right)$} \\
\hline SPDH & $10,25 \mathrm{Aa}$ & $6,55 \mathrm{Ab}$ \\
\hline SPC & $6,67 \mathrm{Ba}$ & $3,52 \mathrm{Bb}$ \\
\hline $\mathrm{PA}$ & $3,90 \mathrm{Ba}$ & $3,65 \mathrm{Bb}$ \\
\hline $\mathrm{CE}$ & $8,15 \mathrm{Aa}$ & $5,38 \mathrm{Ab}$ \\
\hline \multirow[t]{2}{*}{$\mathrm{CV}(\%)$} & $15,0^{(2)}$ & $20,0^{(3)}$ \\
\hline & \multicolumn{2}{|c|}{$\operatorname{COT}\left(\mathrm{g} \mathrm{kg}^{-1}\right)$} \\
\hline SPDH & $25,94 \mathrm{Aa}$ & $18,39 \mathrm{Ab}$ \\
\hline SPC & $16,14 \mathrm{Ba}$ & $16,34 \mathrm{Ba}$ \\
\hline $\mathrm{PA}$ & $16,32 \mathrm{Ba}$ & $16,37 \mathrm{Ba}$ \\
\hline $\mathrm{CE}$ & $23,12 \mathrm{Aa}$ & $19,17 \mathrm{Ab}$ \\
\hline \multirow[t]{2}{*}{$\mathrm{CV}(\%)$} & 9,1 & 6,2 \\
\hline & \multicolumn{2}{|c|}{$\mathrm{N}\left(\mathrm{g} \mathrm{kg}^{-1}\right)$} \\
\hline SPDH & $0,88 \mathrm{Aa}$ & $0,54 \mathrm{Ab}$ \\
\hline SPC & $0,62 \mathrm{Ba}$ & $0,67 \mathrm{Aa}$ \\
\hline PA & $0,62 \mathrm{Ba}$ & $0,65 \mathrm{Aa}$ \\
\hline $\mathrm{CE}$ & $0,87 \mathrm{Aa}$ & $0,66 \mathrm{Ab}$ \\
\hline \multirow[t]{2}{*}{$\mathrm{CV}(\%)$} & 13,8 & 13,0 \\
\hline & \multicolumn{2}{|c|}{ Relação C/N } \\
\hline SPDH & $29,65 \mathrm{Aa}$ & $34,01 \mathrm{Aa}$ \\
\hline $\mathrm{SPC}$ & $25,95 \mathrm{Ba}$ & $24,11 \mathrm{Ba}$ \\
\hline PA & $26,51 \mathrm{Ba}$ & $26,06 \mathrm{Ba}$ \\
\hline $\mathrm{CE}$ & $26,69 \mathrm{Ba}$ & $28,99 \mathrm{Ba}$ \\
\hline \multirow[t]{2}{*}{$\mathrm{CV}(\%)$} & & 13,1 \\
\hline & \multicolumn{2}{|c|}{$\operatorname{EstC}\left(\mathrm{Mg} \mathrm{ha}^{-1}\right)$} \\
\hline SPDH & $19,97 \mathrm{Aa}$ & $19,07 \mathrm{Aa}$ \\
\hline SPC & $14,63 \mathrm{Ba}$ & $15,59 \mathrm{Ba}$ \\
\hline PA & $15,08 \mathrm{Ba}$ & $15,64 \mathrm{Ba}$ \\
\hline $\mathrm{CE}$ & $20,87 \mathrm{Aa}$ & $18,80 \mathrm{Aa}$ \\
\hline \multirow[t]{2}{*}{ CV (\%) } & 11,7 & 12,1 \\
\hline & \multicolumn{2}{|c|}{ EstN $\left(\mathrm{Mg} \mathrm{ha}^{-1}\right)$} \\
\hline SPDH & 0,72 & 0,54 \\
\hline SPC & 0,58 & 0,64 \\
\hline PA & 0,57 & 0,56 \\
\hline $\mathrm{CE}$ & 0,77 & 0,64 \\
\hline $\mathrm{CV}(\%)$ & 14,8 & 16,2 \\
\hline \multicolumn{3}{|c|}{$\begin{array}{l}{ }^{(1)} \text { Médias seguidas de letras iguais, minúsculas na comparação entre siste } \\
\text { mas de manejo e maiúsculas na comparação entre profundidades, não di } \\
\text { ferem pelo teste t, a } 5 \% \text { de probabilidade. }{ }^{(2)} \text { Coeficiente de variação }(\mathrm{CV} \\
\text { entre as áreas. }{ }^{(3)} \mathrm{CV} \text { entre as profundidades. MOL, matéria orgânica leve } \\
\mathrm{COT}, \mathrm{C} \text { orgânico total; } \mathrm{N} \text {, teor de nitrogênio; EstC, estoque de C; e EstN } \\
\text { estoque de nitrogênio. }\end{array}$} \\
\hline
\end{tabular}


Os maiores valores de $\mathrm{MOL}, \mathrm{COT}$, $\operatorname{EstC}(0,0-0,10 \mathrm{~m})$ e $\mathrm{N}(0,0-0,05 \mathrm{~m})$ na área de $\mathrm{CE}$, em comparação às áreas de SPC e PA(Tabela 2), decorrem da deposição contínua de serapilheira e da ausência de ação antrópica, o que proporciona um ambiente favorável à manutenção dos teores de MOS (Guareschi et al., 2012a). No entanto, na área de SPDH, os maiores valores podem ter sido decorrentes do não revolvimento do solo e do acúmulo de palhada na superfície. Na literatura, verificam-se resultados semelhantes, em que áreas de SPDH aumentam os teores de MOS (Santos et al., 2012), COT (Loss et al., 2009, 2010a, 2010b; Souza et al., 2014b), N (Loss et al., 2009, 2010a, 2010b) e MOL (Loss et al., 2009), em comparação às áreas de SPC.

Os menores valores de MOL, COT, $\mathrm{N}$ e EstC da área de PA, comparados aos das áreas de CE e SPD (Tabela 2), podem ser justificados pela baixa produtividade da pastagem e pela ausência da correção do solo e de adubações de manutenção. De acordo com Guareschi et al. (2012a), a falta de manejo da fertilidade do solo diminui o aporte de material orgânico no solo, com redução concomitante dos conteúdos de MOL e MOS.

A área de SPDH apresentou maior relação $\mathrm{C} / \mathrm{N}$ do que as demais áreas avaliadas, nas profundidades de 0,0-0,05 e 0,05-0,10 m (Tabela 2), possivelmente em razão do grande aporte de resíduos vegetais $\left(16 \mathrm{Mg} \mathrm{ha}^{-1}\right.$ de matéria seca) deixado pela cultura do milho, antes da implantação do sistema. Essa grande quantidade de resíduos, somada à alta relação $\mathrm{C} / \mathrm{N}$ (64), faz com que a mineralização seja lenta, com reflexos na relação $\mathrm{C} / \mathrm{N}$ do solo.

As maiores quantidades de MOL, COT e N, na camada mais superficial $(0-0,05 \mathrm{~m})$, nas áreas de $\mathrm{CE}$ e SPDH (Tabela 2), foram decorrentes do maior aporte de resíduos vegetais depositados sobre o solo, nessas áreas. Souza et al. (2014b), ao avaliar áreas de SPDH e SPC, também verificaram que as principais alterações dos atributos avaliados ocorreram na camada mais superficial, e que o SPDH destacou-se como o sistema com os maiores teores de COT.

A Ds em áreas de cerrado é naturalmente baixa; portanto, a ação antrópica nas áreas de SPDH, SPC e PA aumentaram os valores desse atributo, na camada de $0,0-0,05 \mathrm{~m}$ (Tabela 3). Quanto à Ds na camada de 0,05-0,10 m, as áreas de SPDH e PA apresentaram valores estatisticamente semelhantes entre si, mas superiores aos das áreas de CE e SPC. Os menores valores de Ds na área de $\mathrm{CE}$, em comparação às áreas de cultivo PA e SPD, podem ser atribuídos ao maior acúmulo de resíduos vegetais e $\mathrm{C}$ na superfície do solo e pela menor alteração antrópica. Já a menor Ds do SPC, na camada $0,05-0,10 \mathrm{~m}$, pode estar relacionada

Tabela 3. Densidade do solo, C orgânico particulado (COp), C orgânico associado aos minerais (COam), ácido fúlvico, ácido húmico e humina, em áreas sob plantio direto de hortaliças (SPDH) e sob plantio convencional (SPC), cultivadas com repolho, pastagem (PA) e sob cerrado nativo (CE), em duas profundidades ${ }^{(1)}$.

\begin{tabular}{|c|c|c|}
\hline \multirow[t]{2}{*}{ Área } & \multicolumn{2}{|c|}{ Profundidade } \\
\hline & $0,0-0,05 \mathrm{~m}$ & $0,05-0,10 \mathrm{~m}$ \\
\hline & \multicolumn{2}{|c|}{ Densidade do solo $\left(\mathrm{Mg} \mathrm{m}^{-3}\right)$} \\
\hline SPDH & $1,25 \mathrm{Aa}$ & $1,26 \mathrm{Aa}$ \\
\hline SPC & $1,21 \mathrm{Aa}$ & $1,05 \mathrm{Ba}$ \\
\hline PA & $1,25 \mathrm{Aa}$ & $1,30 \mathrm{Aa}$ \\
\hline $\mathrm{CE}$ & $1,02 \mathrm{Ba}$ & $1,03 \mathrm{Ba}$ \\
\hline \multirow[t]{2}{*}{$\mathrm{CV}(\%)$} & $7,4^{(2)}$ & $6,4^{(3)}$ \\
\hline & \multicolumn{2}{|c|}{$\operatorname{COp}\left(\mathrm{g} \mathrm{kg}^{-1}\right)$} \\
\hline SPDH & $11,30 \mathrm{Aa}$ & $6,80 \mathrm{Ab}$ \\
\hline SPC & $5,31 \mathrm{Ba}$ & $5,51 \mathrm{Ba}$ \\
\hline PA & $3,96 \mathrm{Ba}$ & $3,28 \mathrm{Ba}$ \\
\hline $\mathrm{CE}$ & $4,67 \mathrm{Ba}$ & $3,31 \mathrm{Ba}$ \\
\hline \multirow[t]{2}{*}{ CV $(\%)$} & $25,8^{(3)}$ & $24,2^{(4)}$ \\
\hline & \multicolumn{2}{|c|}{$\operatorname{Coam}\left(\mathrm{g} \mathrm{kg}^{-1}\right)$} \\
\hline SPDH & $14,63 \mathrm{Ba}$ & $11,58 \mathrm{Ba}$ \\
\hline SPC & $10,82 \mathrm{Ca}$ & $10,83 \mathrm{Ca}$ \\
\hline PA & $12,36 \mathrm{Ba}$ & $13,08 \mathrm{Ba}$ \\
\hline $\mathrm{CE}$ & $18,45 \mathrm{Aa}$ & $15,86 \mathrm{Aa}$ \\
\hline \multirow[t]{2}{*}{ CV $(\%)$} & 11,9 & 11,2 \\
\hline & \multicolumn{2}{|c|}{ Ácido fúlvico $\left(\mathrm{g} \mathrm{kg}^{-1}\right)$} \\
\hline SPDH & 7,92Aa & $6,50 \mathrm{Ab}$ \\
\hline SPC & $6,57 \mathrm{Ba}$ & $6,53 \mathrm{Aa}$ \\
\hline PA & $7,05 \mathrm{Ba}$ & $6,34 \mathrm{Aa}$ \\
\hline $\mathrm{CE}$ & $8,24 \mathrm{Aa}$ & $5,77 \mathrm{Ab}$ \\
\hline \multirow[t]{2}{*}{ CV $(\%)$} & 5,3 & 7,2 \\
\hline & \multicolumn{2}{|c|}{ Ácido húmico $\left(\mathrm{g} \mathrm{kg}^{-1}\right)$} \\
\hline SPDH & $2,86 \mathrm{Aa}$ & $1,62 \mathrm{Bb}$ \\
\hline SPC & $2,41 \mathrm{Ba}$ & $1,43 \mathrm{Bb}$ \\
\hline PA & $1,79 \mathrm{Ba}$ & $1,69 \mathrm{Bb}$ \\
\hline $\mathrm{CE}$ & $3,62 \mathrm{Aa}$ & $2,53 \mathrm{Ab}$ \\
\hline \multirow[t]{2}{*}{$\mathrm{CV}(\%)$} & 16,9 & 15,9 \\
\hline & \multicolumn{2}{|c|}{ Humina $\left(\mathrm{g} \mathrm{kg}^{-1}\right)$} \\
\hline SPDH & $15,15 \mathrm{Aa}$ & $10,27 \mathrm{Ab}$ \\
\hline SPC & $7,14 \mathrm{Ba}$ & $8,37 \mathrm{Ba}$ \\
\hline PA & $7,46 \mathrm{Ba}$ & $8,33 \mathrm{Ba}$ \\
\hline $\mathrm{CE}$ & $11,26 \mathrm{Aa}$ & $10,86 \mathrm{Aa}$ \\
\hline CV (\%) & 20,39 & 12,34 \\
\hline
\end{tabular}

${ }^{(1)}$ Médias seguidas de letras iguais, minúsculas na comparação entre sistemas de manejo e maiúsculas na comparação entre profundidades, não diferem pelo teste t, a $5 \%$ de probabilidade. ${ }^{(2)}$ Coeficiente de variação $(\mathrm{CV})$ entre as áreas. ${ }^{(3)} \mathrm{CV}$ entre as profundidades. 
à mobilização do solo pelo arado, grade ou enxadas rotativas, o que pode ter aliviado temporariamente a compactação causada pelas operações de manejo das culturas. Resultados semelhantes foram observados por Guareschi et al. (2012b) que, ao avaliar sistemas de manejo em um Latossolo Vermelho, também verificaram que áreas de SPC e CE apresentaram menores valores de Ds do que as áreas de PA e SPD.

Os maiores valores de Ds da área de SPDH (Tabela 3) também podem ser explicados pela implantação do SPD que, apesar de recente, pode ter promovido a reorganização de partículas primárias do solo, que são influenciadas pelo aumento dos teores de MOS. Ainda assim, é provável que, com o tempo, os teores de COT se mantenham e que a atividade biológica do solo aumente na área sob SPDH, o que melhoraria ainda mais a agregação do solo, com tendência de redução da Ds (Figueiredo et al., 2008).

Em relação à área de $\mathrm{PA}$, os maiores valores de Ds, em comparação às áreas de CE e SPC (Tabela 3), podem ser consequência do menor conteúdo de $\mathrm{C}$ ou do pastoreio excessivo na área avaliada (Figueiredo et al., 2008; Guareschi et al., 2012b).

Aárea de SPDH apresentou os maiores teores de COp $(0,0-0,10 \mathrm{~m})$ (Tabela 3). Na camada de 0,0-0,05 m, esses teores foram superiores aos da camada de 0,05 $0,10 \mathrm{~m}$. Estes resultados podem estar relacionados ao maior aporte de material orgânico na superfície do solo nessa área. Loss et al. (2009), ao comparar uma área SPD de beringela/milho e SPC milho/feijão, em Argissolo Vermelho-Amarelo, também observaram maiores teores de COp no SPD. Os autores atribuíram estes resultados à maior adição de resíduos vegetais à superfície do solo.

Quanto aos resultados do COam $(0,0-0,10 \mathrm{~m})$, os maiores teores foram observados na área de $\mathrm{CE}$, seguida pelas áreas de SPDH e PA, que não diferiram estatisticamente entre si, e, por fim, da área de SPC (Tabela 3). Mais uma vez, pode-se constatar que a área de CE, por tratar-se de um ambiente não antropizado e com constante aporte de resíduos vegetais, tendeu a apresentar maior estabilidade da matéria orgânica, principalmente pela interação com a fração mineral do solo. Guareschi et al. (2013) avaliaram áreas de SPD, PA e CE, e também observaram esse padrão de resposta, em que a área de $\mathrm{CE}$ apresentou maiores teores de COam do que as demais.
Os menores valores de COam na área de SPC, em comparação às demais áreas (Tabela 3), podem ter sido decorrentes do revolvimento do solo nesse sistema de manejo, que pode acelerar a mineralização da MOS pela ruptura dos agregados e exposição desta à ação dos microrganismos. Portanto, a estabilidade da MOS fica comprometida nesse manejo. No entanto, na área de SPDH, o padrão é inverso, em que o não revolvimento do solo, a rotação de culturas, a adubação orgânica e a manutenção dos resíduos vegetais em superfície contribuem para aumento do conteúdo de COT e promovem sua maior interação com a fase mineral do solo, o que, consequentemente, aumenta a estabilidade dos agregados. Resultados similares foram relatados por Loss et al. (2009), que também verificaram maior teor de COam na área de SPD, em comparação ao SPC.

As áreas de SPDH e CE não diferiram quanto às frações $\mathrm{AF}, \mathrm{AH}(0,0-0,5)$ e HUM $(0,0-0,10 \mathrm{~m}) \mathrm{e}$ apresentaram teores superiores aos das áreas de SPC e PA (Tabela 3). Estes resultados apresentaram o mesmo padrão observado para MOL, COT, EstC $(0,0-0,10 \mathrm{~m})$ e $\mathrm{N}(0,0-0,05 \mathrm{~m})$. Loss et al. (2009) também constataram maiores teores de AF, AH e HUM em áreas de SDP, em comparação às áreas de SPC, e também observaram correlações positivas com os valores de COT. De maneira similar, Souza et al. (2014b) verificaram maiores teores de HUM em áreas de SPDH, em comparação ao SPC.

A HUM é a fração que contém a maior parte do COT do solo, seguida por AF e AH (Tabela 3). De acordo com esses resultados, pode-se inferir que o SPDH, mesmo nesta fase inicial de implantação, está em processo acelerado de humificação da MOS, com consequente formação de AF (Loss et al., 2010b). No entanto, como as outras frações (AH e HUM) também aumentaram, é provável que a distribuição do $\mathrm{C}$ nas frações húmicas tenha ocorrido a partir das frações mais lábeis (ácidos fúlvicos) para as mais estáveis (ácidos húmicos e humina). Segundo Guareschi et al. (2013), em áreas de SPD pode ocorrer uma rápida conversão de ácidos fúlvicos em ácidos húmicos. De acordo com Loss et al. (2010b), áreas em SPD, pelo menor revolvimento do solo e, consequentemente, maior preservação dos resíduos vegetais em superfície, o aumento dos teores AH é favorecido com o tempo de adoção do sistema. Os maiores teores de AH do SPDH, em comparação ao SPC, representam um aspecto favorável para a qualidade física do solo, tendo-se em vista que esta 
fração húmica é responsável pela maior capacidade de troca catiônica de origem orgânica, em camadas superficiais de solos (Benites et al., 2003).

Os maiores teores de HUM, nas áreas de CE e SPDH, corroboram os maiores valores observados de COam (Tabela 3) e indicam que estes sistemas estão sendo capazes de estocar $\mathrm{C}$ no solo, por meio da interação organomineral. Maiores valores de C na HUM, em geral, favorecem maior expressão de propriedades da fração coloidal da matéria orgânica, tais como: maior retenção de água e cátions, além melhoria da agregação do solo, que são características de grande importância para a sustentabilidade da produção agrícola (Guareschi et al., 2013).

Os menores teores de $\mathrm{C}$ nas frações húmicas, observados nas áreas de PA e SPC (Tabela 3), estão relacionados aos seus históricos de uso. No caso de PA, a falta de adubação de manutenção, com a consequente baixa produtividade, diminui o aporte de material orgânico no solo, o que contribui para a redução dos teores de $\mathrm{C}$ das frações húmicas. A mobilização do solo no SPC favorece a decomposição da MOS, pela maior aeração do solo e exposição da matéria orgânica, o que aumenta a atividade microbiana e acelera a degradação da MOS (Loss et al., 2010b).

Em geral, assim como o observado para o COT, os maiores conteúdos das frações húmicas se concentraram na camada de $0,0-0,05 \mathrm{~m}$ (Tabela 3 ). Esse padrão foi relatado também em outros estudos (Loss et al., 2010b; Souza et al., 2014b).

Para as frações oxidáveis, as áreas de CE e SPDH apresentaram os maiores teores de C na F1 $(0,00-10 \mathrm{~m})$, e na F3 e F4 (0,0-0,05 m), em comparação às áreas de PA e SPC (Tabela 4). Assim, verifica-se que o manejo adotado nas áreas de PA e SPC não foi capaz de estocar e manter a estabilidade da MOS, pois os menores valores de $\mathrm{C}$ na fração $\mathrm{F} 1$ indicam redução do $\mathrm{C}$ lábil do solo, enquanto os menores valores nas frações F3 e F4 mostram que as frações mais recalcitrantes ou associadas aos minerais não estão sendo favorecidas. Estes resultados estão relacionados, mais uma vez, ao que foi discutido sobre os históricos de manejo. Resultados semelhantes foram observados por Souza et al. (2014b), que também constataram menores teores de C nas frações F1, no SPC, em comparação ao SPD, e atribuíram estes resultados à influência do revolvimento do solo sobre a degradação do $\mathrm{C}$ orgânico de fácil oxidação. Rangel et al. (2008), Loss et al. (2010a) e Souza et al. (2014b) apontam que os maiores teores de $\mathrm{C}$ nas frações $\mathrm{F} 1$ e $\mathrm{F} 2$ tendem a ser encontrados nas áreas onde há grande aporte de matéria orgânica, resultantes de resíduos vegetais na superfície do solo, e que o aumento dessas frações está relacionado, principalmente, ao acúmulo da matéria orgânica leve da MOS, o que também foi constatado no presente trabalho.

Os maiores teores de $\mathrm{C}$ nas frações F3 e F4 $(0,0$ 0,05 m), nas áreas de CE e SPDH (Tabela 4), mostram, mais uma vez, que estes sistemas são capazes de manter e estocar $\mathrm{C}$ em frações mais resistentes. Loss et al. (2010a) também observaram este padrão e verificaram que áreas com maiores quantidades destas

Tabela 4. Frações oxidáveis da matéria orgânica do solo, em áreas sob plantio direto de hortaliças (SPDH) e sob plantio convencional (SPC), cultivadas com repolho, pastagem (PA) e sob cerrado nativo (CE), em duas profundidades ${ }^{(1)}$.

\begin{tabular}{lcc}
\hline \multirow{2}{*}{ Área } & \multicolumn{2}{c}{ Profundidade } \\
\cline { 2 - 3 } & $0,0-0,05 \mathrm{~m}$ & $0,05-0,10 \mathrm{~m}$ \\
\hline SPDH & \multicolumn{2}{c}{$\mathrm{F} 1\left(\mathrm{~g} \mathrm{~kg}^{-1}\right)$} \\
SPC & $1,30 \mathrm{Aa}$ & $1,12 \mathrm{Ab}$ \\
PA & $1,18 \mathrm{Ba}$ & $1,02 \mathrm{Bb}$ \\
CE & $1,47 \mathrm{Aa}$ & $1,03 \mathrm{Bb}$ \\
\hline CV (\%) & $12,92^{(2)}$ & $1,28 \mathrm{Ab}$ \\
\hline & \multicolumn{2}{c}{$11,64^{(3)}$} \\
SPDH & $0,85 \mathrm{Aa}$ & $0,47 \mathrm{Ab}$ \\
SPC & $0,77 \mathrm{Aa}$ & $0,68 \mathrm{Aa}$ \\
PA & $0,68 \mathrm{Aa}$ & $0,87 \mathrm{Aa}$ \\
CE & $0,87 \mathrm{Aa}$ & $0,81 \mathrm{Aa}$ \\
\hline CV (\%) & 16,5 & 20,08 \\
\hline & & $\mathrm{F} 3\left(\mathrm{~g} \mathrm{~kg}^{-1}\right)$ \\
SPDH & $2,47 \mathrm{Aa}$ & $1,80 \mathrm{Ab}$ \\
SPC & $1,79 \mathrm{Ca}$ & $1,64 \mathrm{Aa}$ \\
PA & $2,07 \mathrm{Ba}$ & $2,21 \mathrm{Aa}$ \\
CE & $2,38 \mathrm{Aa}$ & $1,73 \mathrm{Ab}$ \\
\hline CV (\%) & 6,73 & 15,8 \\
\hline & & $\mathrm{F} 4\left(\mathrm{~g} \mathrm{~kg}^{-1}\right)$ \\
SPDH & $2,90 \mathrm{Aa}$ & $2,00 \mathrm{Ab}$ \\
SPC & $2,14 \mathrm{Ca}$ & $2,14 \mathrm{Aa}$ \\
PA & $2,22 \mathrm{Ca}$ & $2,10 \mathrm{Aa}$ \\
CE & $2,58 \mathrm{Ba}$ & $2,21 \mathrm{Ab}$ \\
\hline CV (\%) & 2,7 & 14,4 \\
\hline & &
\end{tabular}

${ }^{(1)}$ Médias seguidas de letras iguais, minúsculas na comparação entre sistemas de manejo e maiúsculas na comparação entre profundidades, não diferem pelo teste t, a $5 \%$ de probabilidade. ${ }^{(2)}$ Coeficiente de variação $(\mathrm{CV})$ entre as áreas. ${ }^{(3)} \mathrm{CV}$ entre as profundidades. $\mathrm{F} 1$, fração 1 , com $\mathrm{C}$ oxidado por $\mathrm{K}_{2} \mathrm{Cr}_{2} \mathrm{O}_{7}$, em meio ácido a $3 \mathrm{~mol} \mathrm{~L}^{-1} \mathrm{de}_{2} \mathrm{SO}_{4} ; \mathrm{F} 2$, fração 2, obtida pela diferença entre o $\mathrm{C}$ oxidado a 6 e a $3 \mathrm{~mol} \mathrm{~L}^{-1} \mathrm{de}_{2} \mathrm{SO}_{4}$; $\mathrm{F} 3$, fração 3, obtida pela diferença entre o C oxidado a 9 e a $6 \mathrm{~mol} \mathrm{~L}^{-1}$; e F4, fração 4 , obtida pela diferença entre o C oxidado a 12 e a $9 \mathrm{~mol} \mathrm{~L}^{-1}$.

Pesq. agropec. bras., Brasília, v.51, n.9, p.1511-1519, set. 2016 DOI: $10.1590 / \mathrm{S} 0100-204 \mathrm{X} 2016000900050$ 
frações apresentaram maior proteção física e química da MOS.

Em geral, conforme observado para a maioria das variáveis, constatou-se que as áreas que proporcionaram maior teor de $\mathrm{C}$ nas frações oxidáveis também apresentaram maiores teores deste atributo na camada de $0,0-0,05 \mathrm{~m}$, em comparação à camada de $0,05-0,10 \mathrm{~m}$ (Tabela 4). Este padrão também foi constatado em outros trabalhos (Loss et al., 2010a; Souza et al., 2014b) e está relacionado ao maior contato da camada superficial do solo com o material orgânico.

Novos estudos serão importantes para a avaliação dos efeitos do tempo de consolidação do SPD sobre a dinâmica da MOS, bem como de sua influência sobre a fertilidade do solo e a produtividade das culturas. Além disso, esses estudos devem prover informações sobre a melhor maneira de condução do SPDH em diferentes condições edafoclimáticas.

\section{Conclusões}

1. O sistema plantio direto para a cultura do repolho, mesmo em fase inicial de implantação, é capaz de interferir na dinâmica e manutenção da matéria orgânica do solo e aumentar os valores de matéria orgânica leve, $\mathrm{C}, \mathrm{N}$, estoque de $\mathrm{C}, \mathrm{C}$ orgânico particulado e associado a minerais, frações húmicas e oxidáveis, em comparação ao sistema de plantio convencional e à pastagem.

2. O sistema plantio direto melhora a qualidade do solo, em relação ao plantio convencional, além de proporcionar, para a maioria das variáveis analisadas, valores maiores do que os observados em área de pastagem, e valores semelhantes aos da área sob Cerrado nativo.

\section{Referências}

ANDERSON, J.N.; INGRAM, J.S.I. Tropical soil biology and fertility: a handbook of methods. Wallingford: CAB International, 1989. 171p.

BENITES, V.M.; MADARI, B.; MACHADO, P.L.O.A. Extração e fracionamento quantitativo de substâncias húmicas do solo: um procedimento simplificado de baixo custo. Rio de Janeiro: Embrapa Solos, 2003. 7p. (Embrapa Solos. Comunicado técnico, 16).

BEUTLER, S.J.; PEREIRA, M.G.; LOSS, A.; PERIN, A.; ANJOS, L.H.C. Humic substances and phosphorus fractions in areas with crop-livestock integration. pasture and natural cerrado vegetation in Goiás, Brazil. Tropical and Subtropical Agroecosystems, v.18, p.11-25, 2015.

BIANCHINI, C. Sistemas de manejo do solo para a produção de abobrinha de tronco (Curcubita pepo). 2013. 79p. Dissertação (Mestrado em Agronomia) - Universidade Tecnológica Federal do Paraná, Pato Branco.

CAMBARDELLA, C.A.; ELLIOTT, E.T. Methods for physical separation and characterization of soil organic matter fractions. Geoderma, v.56, p.449-457, 1993. DOI: 10.1016/00167061(93)90126-6.

CHAN, K.Y.; BOWMAN, A.; OATES, A. Oxidizible organic carbon fractions and soil quality changes in an Paleustalf under different pasture leys. Soil Science, v.166, p.61-67, 2001. DOI: 10.1097/00010694-200101000-00009.

DONAGEMMA, G.K.; CAMPOS, D.V.B. de; CALDERANO, S.B.; TEIXEIRA, W.G.; VIANA, J.H.M. (Org.). Manual de métodos de análise de solo. 2.ed. ver. Rio de Janeiro: Embrapa Solos, 2011. 230p. (Embrapa Solos. Documentos, 132).

FIGUEIREDO, C.C.; RAMOS, M.L.G.; TOSTES, R. Propriedades físicas e matéria orgânica de um Latossolo Vermelho sob sistemas de manejo e cerrado nativo. Bioscience Journal, v.24, p.24-30, 2008.

GALVÃO, R. de O.; ARAÚJO NETO, S.E. de; FERREIRA, R.L.F. Plantio direto orgânico de alface sobre cobertura viva e morta e adubada com composto. Agropecuária Científica no Semiárido, v.9, p.75-80, 2013.

GUARESCHI, R.F.; PEREIRA, M.G.; PERIN, A. Deposição de resíduos vegetais, matéria orgânica leve, estoques de carbono e nitrogênio e fósforo remanescente sob diferentes sistemas de manejo no Cerrado goiano. Revista Brasileira de Ciência do Solo, v.36, p.909-920, 2012a. DOI: 10.1590/S0100-06832012000300021.

GUARESCHI, R.F.; PEREIRA, M.G.; PERIN, A. Estoque de carbono em Latossolo Vermelho distroférrico sob diferentes sistemas de manejo. Revista Brasileira de Ciências Agrárias, v.7, p.597-602, 2012b. DOI: 10.5039/agraria.v7i4a1767.

GUARESCHI, R.F.; PEREIRA, M.G.; PERIN, A. Frações da matéria orgânica em áreas de Latossolo sob diferentes sistemas de manejo no Cerrado do Estado de Goiás. Semina: Ciências agrárias, v.34, p.2615-2628, 2013.

LEITE, L.F.C.; GALVÃO, S.R.S.; HOLANDA NETO, M.R.; ARAÚJO, F.C.; IWATA, B.F. Atributos químicos e estoques de carbono em Latossolo sob plantio direto no Cerrado do Piauí. Revista Brasileira de Engenharia Agrícola e Ambiental, v.14, p.1273-1280, 2010. DOI: 10.1590/S1415-43662010001200004.

LOSS, A.; MORAES, A.G. de L.; PEREIRA, M.G.; SILVA, E.M.R. da; ANJOS, L.H.C. dos. Carbono, matéria orgânica leve e frações oxidáveis do carbono orgânico sob diferentes sistemas de produção orgânica. Comunicata Scientiae, v.1, p.57-64, 2010a.

LOSS, A.; PEREIRA, M.G.; PERIN, A.; BEUTLER, S.J.; ANJOS, L.H.C. Carbon, nitrogen and natural abundance of $\delta^{13} \mathrm{C}$ e $\delta^{15} \mathrm{~N}$ of light-fraction organic matter under no-tillage and crop-livestock integration systems. Acta Scientiarum. Agronomy, v.34, p.465472, 2012. DOI: 10.4025 /actasciagron.v34i4.15061. 
LOSS, A.; PEREIRA, M.G.; SCHULTZ, N.; ANJOS, L.H.C. dos; SILVA, E.M.R. da. Carbono e frações granulométricas da matéria orgânica do solo sob sistemas de produção orgânica. Ciência Rural, v.39, p.1067-1072, 2009. DOI: 10.1590/S010384782009005000036 .

LOSS, A.; PEREIRA, M.G.; SCHULTZ, N.; ANJOS, L.H.C. dos; SILVA, E.M.R. da. Quantificação do carbono das substâncias húmicas em diferentes sistemas de uso do solo e épocas de avaliação. Bragantia, v.69, p.913-922, 2010b. DOI: 10.1590/ S0006-87052010000400018.

NEVES, J.F.; SILVA, L.B.; SEABRA JÚNIOR, S.; NEVES, S.M.A. da S.; NEVES, R.J.; DASSOLLER, T.F. O cultivo de hortaliças sobre plantio direto e coberturas de solo em Cáceres/ MT. Cadernos de Agroecologia, v.9, p.1-8, 2014.

PAULETTI, D.R. Influência das plantas de cobertura nas características produtivas da alface e nos atributos físicos e químicos do solo. 2012. 81p. Dissertação (Mestrado) Universidade Estadual do Oeste do Paraná, Marechal Candido Rondom.

PEREIRA, M.F.S.; NOVO JÚNIOR, J.; SÁ, J.R. de; LINHARES, P.C.F.; BEZERRA NETO, F.; PINTO, J.R. de S. Ciclagem do carbono do solo nos sistemas de plantio direto e convencional. Agropecuária Científica no Semiárido, v.9, p.21-32, 2013.

PEREIRA, M.G.; LOSS, A.; BEUTLER, S.J.; TORRES, J.L. Carbono, matéria orgânica leve e fósforo remanescente em diferentes sistemas de manejo do solo. Pesquisa Agropecuária Brasileira, v.45, p.508-514, 2010. DOI: 10.1590/S0100204X2010000500010.

RAMOS, M.R.; FAVARETTO, N.; UHLMANN, U.; DIECKOW, J.; VEZZANI, F.; ALMEIDA, L. Produção de hortaliças no sistema orgânico: efeito nos atributos físicos do solo. Revista de Ciências Agrárias, v.58, p.45-51, 2015. DOI: 10.4322/rca.1666.

RANGEL, O.J.P.; SILVA, C.A.; GUIMARÃES, P.T.G.; GUILHERME, L.R.G. Frações oxidáveis do carbono orgânico de Latossolo cultivado com cafeeiro em diferentes espaçamentos de plantio. Ciência e Agrotecnologia, v.32, p.429-437, 2008. DOI: 10.1590/S1413-70542008000200013.
SANTOS, H.P. dos; FONTANELI, R.S.; FONTANELI, R.S.; TOMM, G.O. Leguminosas forrageiras anuais de inverno. In: FONTANELI, R.S; SANTOS, H.P. dos; FONTANELI, R.S. (Ed.). Forrageiras para integração lavoura-pecuária-floresta na região sul-brasileira. 2.ed. Brasília: Embrapa, 2012. p.305320.

SILVA, F. de F. da; FREDDI, O. da S.; CENTURION, J.F.; ARATANI, R.G.; ANDRIOLI, F.F.; ANDRIOLI, I. Propriedades físicas de um Latossolo Vermelho cultivado no sistema plantio direto. Irriga, v.13, p.191-204, 2008.

SIQUEIRA NETO, M.; SCOPEL, E.; CORBEELS, M.; CARDOSO, A.N.; DOUZET, J.-M.; FELLER, C.; PICCOLO, M. de C.; CERRI, C.C.; BERNOUX, M. Soil carbon stocks under notillage mulch-based cropping systems in the Brazilian Cerrado: an on-farm synchronic assessment. Soil and Tillage Research, v.110, p.187-195, 2010. DOI: 10.1016/j.still.2010.07.010.

SISTI, C.P.J.; SANTOS, H.P. dos; KOHHANN, R.; ALVES, B.J.R.; URQUIAGA, S.; BODDEY, R.M. Change in carbon and nitrogen stocks in soil under 13 years of conventional or zero tillage in southern Brazil. Soil and Tillage Research, v.76, p.3958, 2004. DOI: 10.1016/j.still.2003.08.007.

SOUZA, J.L. de; GUIMARÃES, G.P. Rendimento de massa de adubos verdes e o impacto na fertilidade do solo em sucessão de cultivos orgânicos. Bioscience Journal, v.29, p.1796-1805, 2013.

SOUZA, R.F. de; MADEIRA, N.R.; FIGUEIREDO, C.C. de. Perdas de solo, água e nutrientes em área cultivada com hortaliças sob sistema de plantio direto. Revista Científica, v.1, p.38-50, 2014a.

SOUZA, R.F. de; FIGUEIREDO, C.C. de; MADEIRA, N.R.; ALCÂNTARA, F.A. de. Effect of management systems and cover crops on organic matter dynamics of soil under vegetables. Revista Brasileira de Ciência do Solo, v.38, p.923-933, 2014b. DOI: 10.1590/S0100-06832014000300024.

YEOMANS, J.C.; BREMNER, J.M. A rapid and precise method for routine determination of organic carbon in soil. Communications in Soil, Science and Plant Analysis, v.19, p.1467-1476, 1988. DOI: 10.1080/00103628809368027.

$\overline{\text { Recebido em } 23 \text { de agosto de } 2015 \text { e aprovado em } 14 \text { de dezembro de } 2015}$ 